\title{
THE EFFECT OF POLARIZED POLYCHROMATIC NON- COHERENT LIGHT (BIOPTRON) THERAPY ON PATIENTS WITH LOWER BACK PAIN
}

\author{
Mariyana Mihaylova ${ }^{1}$, Zhenya Ruseva $^{2}$, Silviya Filkova ${ }^{3}$
}

${ }^{1}$ Department of Physiotherapy, Rehabilitation, Thalassotherapy, Occupational Diseases

and Disaster Medicine, Faculty of Public Health, Medical University of Varna

${ }^{2}$ Department of General Medicine and Clinical Laboratory, Faculty of Medicine, Medical University of Varna

${ }^{3}$ Training and Research Sector of Rehabilitation Therapist, Medical College, Medical University of Varna

\section{ABSTRACT}

INTRODUCTION: Lately the polarized polychromatic non-coherent lower energy light emitted by the Bioptron lamp has become more widely used in the physiotherapeutic practice.

AIM: To study the effect of polarized polychromatic incoherent low energy light on pain, motor activity and quality of life in patients with vertebrogenic lower back pain and to compare it with other methods used in practice.

MATERIALS AND METHODS: Object of the study were 60 patients with lower back pain. A control group $(\mathrm{N}=30$, age $46 \pm 11.5 \mathrm{y}$.) was treated with basic therapy and the Bioptron apparatus. Functional tests of the motor activity (test of Thomayer and test of Schober), evaluation of the pain using visual analogue scale (VAS) evaluation of the quality of life using the Roland-Morris test in the beginning, at the end and 1 month after the treatment were used to evaluate the effectiveness of treatment.

RESULTS: The results have a statistical significance of $(p<0.05)$ for both groups regarding the reduction of the pain syndrome, the functional tests for mobility in the lumbar region and the quality of life. There's also a statistical significance in comparing the results of the test group (TG) and the control group (CG) $(p<0.05)$.

CONCLUSION: Both methods reduced pain, improved the mobility of the lumbar region and improved the quality of life of patients with lower back pain. The complex methodology incorporating basic treatment with the use of polarized polychromatic non-coherent low energy light yielded better results than just basic treatment.

Keywords: lower back pain, physical therapy, polarized polychromatic non-coherent low energy light (PPL)

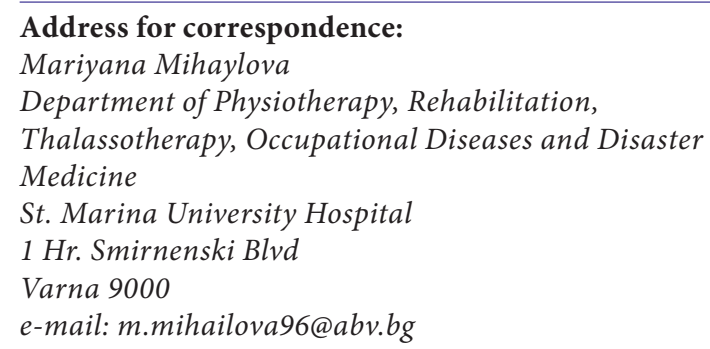

Received: March 31, 2017

Accepted: April 12, 2017 


\section{INTRODUCTION}

The polarized polychromatic non-coherent low energy light (PPL), emitted by the Bioptron apparatus causes warming of the skin because it contains infrared light. This exogenic light is interpreted as irritation by the thermoreceptors and leads to an activation of segment-reflexive and local reactions, improves microcirculation and the trophics of the exposed tissues, has an anti-inflammatory effect. The light changes the sensitivity of the skin, it raises the tactile sensitivity and lowers the pain sensitivity. These effects are confirmed by Ballyzek et al., who studied the change in the amount of unspecified pain in the neck and lower back when treated with PPL. They believe that change of the sensitivity of the receptors, hydrolysis of the products of exchange, lowering of the muscle tonus and increase of the elasticity of the tissue play a role in the relief of pain (10).

Lower back pain is one of the most common musculoskeletal diseases in the world and has major economic effects in industrial countries where at least $80 \%$ of the population experience lower back pain at least once in their life (12). It is one of the most common reasons patients seek hospital care $(8,9,11)$. This fact is connected to the specifics of the biomechanics of the lumbosacral section of the spine $(2,5)$. Considering the important role, the spine plays in our everyday life, its complete functional recovery is an important priority for the medical specialists. The recovery of the impacted static and dynamic function of the spine cannot be achieved solely through drug treatment $(1,4,6)$. The search for new therapeutic approaches to vertebrogenic pain is an important moment in the physiotherapeutic practice. The abovementioned therapeutic effects of PPL, support its inclusion in the physical program of patients with lower back pain (LBP) $(7,10)$.

\section{AIM}

The aim of this study is to study the effectiveness of complex application of polarized polychromatic incoherent low energy light, low frequency magnetic field (LFMF) and ultrasound and compare to that of just LFMF and ultrasound in patients with LBP.

\section{MATERIALS AND METHODS}

60 people with disc disease participated in the study. All of them were patients of the Department of Physiotherapy and Rehabilitation at the St. Marina University Hospital, Varna in the period June - December 2016. The group consisted of 33 men and 27 women. The age of the patients was between 18 and 55 . The experimental group $(48 \pm 11.5)$ consisted of

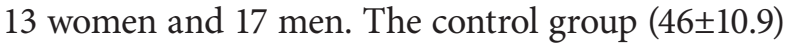
consisted of 14 women and 16 men. Every patient signed a document, giving their informed consent, before being included in the research.

Basic criteria for inclusion of the patients in the study in both groups were:

$\diamond$ age $18-55$ years,

$\diamond$ suffered from lower back pain,

$\diamond$ image diagnostics of the waist region of the spine,

$\diamond$ people, who hadn't had surgery.

Criteria for exclusion from the study:

$\diamond$ patients under 18 years,

$\diamond$ patients with a pacemaker, neoplastic, infections among others,

$\diamond$ disease or condition that wouldn't allow physiotherapy to be performed;

$\diamond$ presence of indications of serious spinal pathology such as tumors, compression fractures, infections, advanced arthrosis (bone bridges) and others;

$\diamond$ paralysis and paresis,

$\diamond$ pregnancy,

$\diamond$ rhythmic pathology,

$\diamond$ surgery in the wait region of the spine,

$\diamond$ neurologic symptoms.

All patients received basic treatment, including LFMF and ultrasound. The first group received only basic treatment and the second was also treated with PPL from the Bioptron apparatus. The treatment was carried out in two parts, paravertebrally, and $10 \mathrm{~min}$. in the waist region.

The patients were randomly separated into two groups. The control group (CG) consisting of $30 \mathrm{pa}-$ tients was treated with basic therapy (LFMF and ultrasound), and the test group (TG) consisting of 30 patients received basic therapy and treatment form 
Mariyana Mihaylova, Zhenya Ruseva, Silviya Filkova

the Bioptron apparatus. In the TG, the physical program was carried out once a day in the following way:

$\diamond$ LFMF with characteristics $16000 \mathrm{~A} / \mathrm{m} .1 \mathrm{~Hz}$, $0.2 \mathrm{~s}, 15-20 \mathrm{~min}$.;

$\diamond$ paravertebral ultrasound treatment of the lumbar portion of the spine from L1 to S1 with parameter $0.4 \mathrm{~W} / \mathrm{cm}, 5 \mathrm{~min}$ on each side,

$\diamond$ treatment with light from the Bioptron apparatus $10 \mathrm{~min} .+10 \mathrm{~min}$. paravertebrally in the lumbar region

Clinical methods were used to track the effects of the treatment: functional test for motor activity (3):

$\diamond$ test of Thomayer - anteflexion (the distance fingers / floor, in cm.);

$\diamond$ sample of Schober - flexion in the lumbar region.

The above-mentioned test was conducted three times - in the beginning, at the end and one month after treatment. Visual analogue scale (VAS) was used for a subjective evaluation of the pain, before and after treatment. The changes in the quality of life were assed using the Roland-Morris questionnaire in the same way.

\section{STATISTICS}

The gathered data was processed through the statistical software IBM SPSS for Windows, v. 19.0 using the following statistical methods:

Descriptive statistics:

$\diamond$ Frequency analysis of the quality variables calculation of absolute and relative frequencies.

$\diamond$ Variation analysis of the quantitative variables - calculation of mean, standard deviation, standard error of the mean, 95\% confidence level.

$\diamond$ Graphic method to visualize the results.

For statistically credible are accepted differences, with a significance level $\mathrm{p}<0.05$.

\section{RESULTS}

The study included 60 patients, whose age ranged between 18 and 55 years. The men among them were 33 and the women - 27. The average age of the test group was $48 \pm 11.5$, while that of the control group was $46 \pm 10.9$. After analyzing the results regarding the pain, measured with VAS, a positive change can be observed in both groups with a significance level $\mathrm{p}<0.05$.

Statistical significance was established in the results of both groups with the test group being superior $(\mathrm{p}<0.05)$ (Fig. 1).

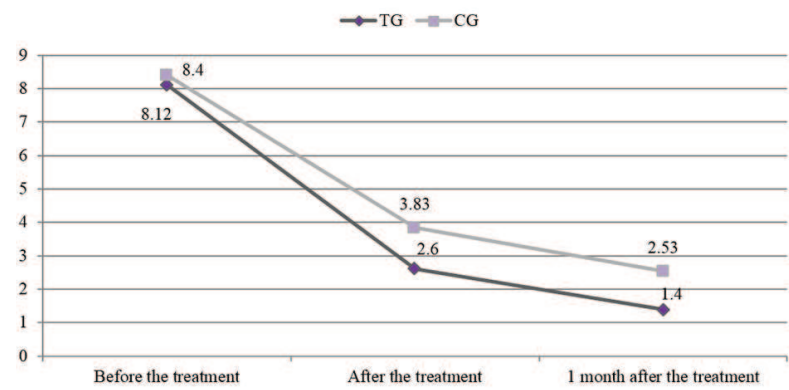

Fig. 1. Pain measured using VAS (in $\mathrm{cm}$ ) before and after treatment and 1 month later

The results from the test of Schober again show a tendency of better results in the TG patients. The mean of the test of Schober has increased with 1.82 $\mathrm{cm}$ from the initial test of the TG and with $1.22 \mathrm{~cm}$ from the initial test of the CG $(\mathrm{p}<0.05)$. This result confirms better mobility of the waist region in the TG patients (Fig. 2).

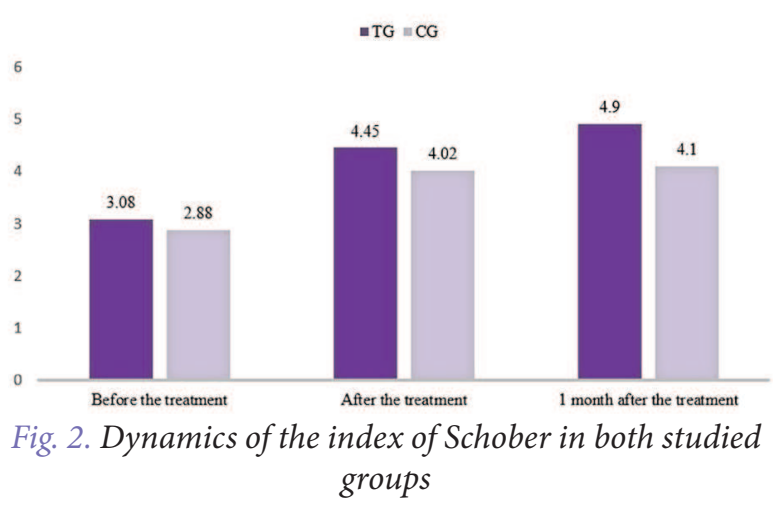

The results from the Thomayer test show a statistically notable increase in the scores of both groups $(\mathrm{p}<0.05)$. In the TG patients, the declination of the body has increased by $10.68 \mathrm{~cm}$ and by 5.78 in the CG ones. The increase in the mobility of the patients in the TG is $4.9 \mathrm{~cm}$ higher than that of the CG and this difference is statistically significant $(\mathrm{p}<0.05)$ (Fig. 3).

The results from the evaluation of the quality of life in both tested groups per the Roland-Mor- 


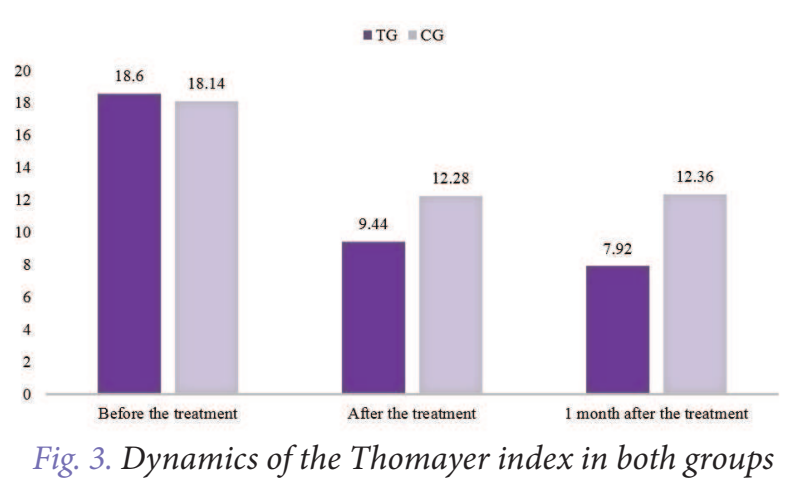

ris questionnaire, show that better quality of life is achieved in patients from the TG. The analysis of the results from the Roland-Morris questionnaire regarding quality of life with lower back pain also confirmed better scores in the TG (Fig. 4).

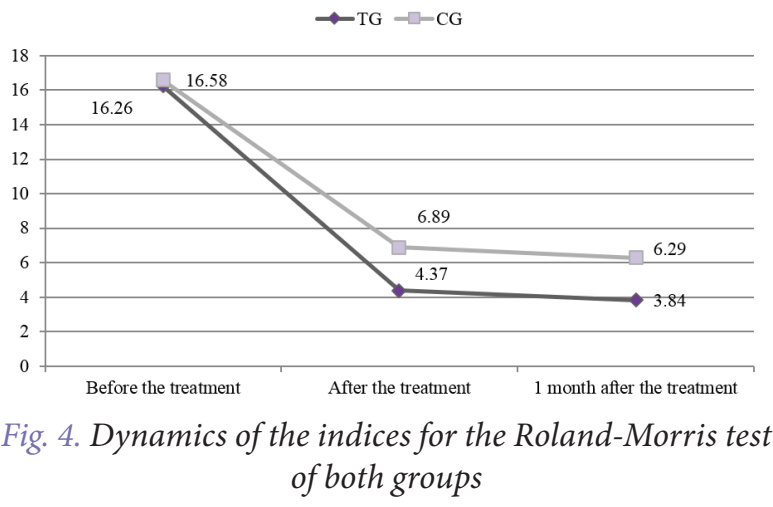

\section{DISCUSSION}

Lower back pain is a serious medico-social issue (12). The research of new methods, supporting the fast and steady recovery of the lost function and the returning of the patient to regular lifestyle is very important. Both of the methodologies that we used have a positive effect on the patient's condition. However, the test group showed superior results in all tracked indicators. The received results regarding the pain, measured through VAS, demonstrate better recovery and reduction of the pain immediately after and preservation of the effect 1 month after the therapy in the test group $\mathrm{p}<0.05$. This ascertainment supports the addition of PPL to the complex of conventional factors for treating the vertebrogenic pain.

The analysis of the results tracking a change of the motor activity through Schober's and Thomay- er's tests, unambiguously supports and proves the superior therapeutic efficiency of the test method. The following changes in the quality of life, tracked through the Ronald-Morris questionnaire before, after and 1 month after the treatment show a positive change in both of the examined groups with an advantage for the test group, $\mathrm{p}<0.05$. The results of our complex methodology with PPL are encouraging, because the presence of infrared light in itself causes warming of the skin, which is perceived as an irritation by the thermoreceptors and leads to activation of the segmental reflective and area reactions, increases the microcirculation and trophics of the irradiated flesh (7). As a result, myorelaxation and pain reduction occur. Under its influence the sensitivity of the skin changes, as well as the tactile sensitivity and reduction of the pain sensitivity is achieved. The positive results of our clinical treatment over the efficiency of the PPL, regarding vertebrogenic pain, support the results cited from Ballyzek et al (10). In their study the cited authors reach the conclusion that pain reduction is achieved through change in the sensitivity of the receptors, hydrolysis of the metabolism products, decrease in the muscle tone and increase in the tissue sensitivity. The superior effect of the methodology using PPL compared to the basic therapy, found in our research as well, proves that PPL has a supplementary effect and contributes to the more significant and lasting influence of the pain syndrome as well as the tracked indicators of motor activity $(\mathrm{p}<0.05)$, and the quality of life $(\mathrm{p}<0.05)$. These results give us a reason to conclude that the reported superior positive changes in the tracked indicators of the test group are because of the specific effect of PPL.

\section{CONCLUSION}

Both methods reduced pain, improved the mobility of the lumbar region and improved the quality of life of patients with lower back pain. The complex methodology incorporating basic treatment with the use of polarized polychromatic non-coherent low energy light yielded better results than just basic treatment. The obtained results prove that the addition of PPL in the complex physical factors for treatment of the lower back pain is justified. This method of treatment is an effective addition to the already existing physical means used in the treatment of vertebrogen- 
ic pain. The results that we obtained are encouraging and give us a reason to continue our studies in that direction.

\section{REFERENCES}

1. Gatev S, Bankov S, Busarov S. Manual of Physical therapy. Sofia: Medicine and physical education; 1992. p. 83-176 (in Bulgarian).

2. Gechev Y. Foundations of general vertebrology. Sofia: Prof. Marin Drinov; 2002 (in Bulgarian).

3. Wolfgang H, Debrunner H. Orthopaedic Diseases. Sofia: Medicine and physical education; 2010. $\mathrm{p}$ 97-8 (in Bulgarian).

4. Kostadinov D, Nikolova L, Bankov S, Slunchev P. Physical factors in the complex treatment and rehabilitation of some common diseases. Sofia: Medicine and physical education; 1975 (in Bulgarian).

5. Milanov I. Neurology: Textbook. Sofia: Medicine and physical education; 2012. p. 78-147 (in Bulgarian).

6. Ryazkova M, Kirova I. Physical therapy - general and specific part. ARSO; 2002 (in Bulgarian).

7. Samoilova KA. Anti-inflammatory, immunomodulatory, stimulating wound healing and normalizing metabolism mechanisms of impact of the light emitted from the apparatus Bioptron. Materials of scientific conference „New directions in the appliance of light treatment with Biotron", MoscowYekaterinburg. 2003: 10-14 (in Russian).

8. Andersson GBJ. The epidemiology of spinal disorders. In: Frymoyer JW (ed.). The adult spine: principles and practice. Philadelphia: Lippincott-Raven; 1997. pp. 93-141.

9. Balagué $\mathrm{F}$, Mannion AF, Pellisé F, Cedraschi C. Non-specific low back pain. Lancet. 2011;379(9814): 482-91. doi: 10.1016/S0140-6736(11)60610-7

10. Ballyzek M, Vesovic-Potic V, He X, Johnston A. Efficacy of polarized, polychromatic, non-coherent light in the treatment of chronic musculoskeletal neck and shoulder pain. Unpublished material, BIOPTRON AG, Wollerau, Switzerland (2005).

11. Battie MC \& Videman, T. Lumbar disc degeneration: epidemiology and genetics. J Bone Joint Surg. Am. 2006; 88 (Suppl. 2), 3-9. doi: 10.2106/ JBJS.E.01313

12. Rubin DI. Epidemiology and risk factors for spine pain. Neurol Clin. 2007. 25(2):353-71. doi: 10.1016/j. ncl.2007.01.004 